УДК 622.279:681.5

\title{
ИДЕНТИФИКАЦИЯ ГИДРОДИНАМИЧЕСКОЙ МОДЕЛИ СКВАЖИНЫ С ЭЛЕКТРОЦЕНТРОБЕЖНЫМ НАСОСОМ ПО ДАННЫМ КОНТРОЛЯ ВОЗМУЩЁННЫХ РЕЖИМОВ ЭКСПЛУАТАЦИИ
}

\author{
Соловьев Илья Георгиевич1,2, \\ solovyev@ikz.ru
}

\author{
Говорков Денис Александрович1,2, \\ dagovorkov@mail.ru \\ Цибульский Владимир Романович1, \\ v-tsib@yandex.ru \\ 1 Федеральный исследовательский центр Тюменский научный центр СО РАН, \\ Россия, 625026, г. Тюмень, ул. Малыгина, 86. \\ 2 Тюменский индустриальный университет, \\ Россия, 625000, г. Тюмень, ул. Володарского, 38.
}

\begin{abstract}
Актуальность исследования связана с проблемами создания и сопровождения динамических моделей скважин с электроцентробежным насосом в контурах оперативного контроля и управления состоянием систем, в условиях дрейфра параметров подъёмника и притока. Оиенка первопричин нарушения предписанного технологического режима работы скважины и прогноз ближайших негативных последствий имеют важное значение для принятия взвешенных (оптимальных) решений по коррекции режимных состояний и обеспечению стабильных условий эксплуатации.

Цель: разработка алгоритма оценивания дрейфующих параметров подъёмника, включая электроцентробежный насос, по методу наименьших квадратов и притока динамической модели скважины и приёмов стабилизации оценок в условиях реальной эксплуатации по данным зашумлённых измерений.

Методы: численное интегрирование нелинейных диффреренциальных уравнений, методы регрессионного анализа по схеме наименьших квадратов, статистических вычислительных испытаний, пробных возмущений, приёмов регуляризации алгоритмов оценивания.

Результаты. Разработан двухэтапный алгоритм оценивания дрейфующих параметров подъёмника (износы, засорения) и притока (параметры продуктивности и подпора пласта) для упрощенного представления комплексной динамической модели скважины класса: «приток-подъёмник- электроцентробежный насос-устье» по данным двухтемповой шкалы контроля режимных состояний системы с реализуемым наблюдателем динамики притока в условиях пробных частотных возмущений.

Выводы. Предложенные правила стабилизации оценок параметров модели на основе генерации пробных возмущений, снижения размерности за счет декомпозиции комплексной модели на составные блоки, фрильтрации зашумлённых сигналов измерений посредством аппроксимации целыми функциями, реализации динамического наблюдателя притока формируют основу конструкции алгоритма идентисикации с надёжным оцениванием основной группы параметров деформации напорной характеристики насоса и притока. Несмотря на низкие показатели точности идентификации гидросопротивления подъёмника, ошибка воспроизведения динамики переменных состояний системы возмущённых режимов эксплуатации с выходов наблюдателя не превышает 2 \%, что говорит о низкой чувствительности итатных режимов эксплуатации подъёмника в рамках используемых модельных решений к искомой настройке параметра. Требуемая точность идентисикации гидросопротивления трубы должна соотноситься с условиями регламента периодической очистки подъёмника от сорбентов.
\end{abstract}

\section{Ключевые слова:}

Скважина, электроцентробежный насос, осложняющие фракторы, декомпозиция, регуляризация, оценки, идентификация, метод наименьших квадратов, наблюдатели, фрильтры состояния, пробные возмущения.

В работе рассматривается развитие задач по построению и моделированию динамики переходных процессов в скважине с регулируемым электроцентробежным насосом (ЭЦН) [1-5]. Речь пойдет о конструировании алгоритма идентификации модели, приведенной ранее в статье [6], по обновляемым данным контроля ее режимных состояний. Заметим, что даже в условиях регламентированных испытаний по оценке параметров притока [7] проблема надёжности и качества оценивания $[8,9]$ всегда остаётся первостепенной. В рассматриваемом случае проблема корректности ещё более усугубляется, т. к. размерность вектора оценок удваивается и помимо притока одно- временно идентифицируются параметры подъёмника, которые в процессе эксплуатации могут дрейфовать. Заметим сразу, что проблема идентификации нестационарных систем в работе не исследуется, так как скорости дрейфов параметров модели много меньше гидродинамики переходных процессов скважины. По сути, нижеизложенное есть обоснование регламента оценивания $[10,11]$ параметров модели скважины по данным непрерывного контроля «слабо» возмущённых режимов нормальной эксплуатации. Как и в классическом анализе, качество оценок напрямую зависит от точности первичного контроля её состояний $[12,13]$. 
Отметим ряд важных моментов.

1. Исходная гидродинамическая модель, ориентированная на задачи оперативного контроля и управления режимами эксплуатации скважин, учитывая взаимодействие главных компонент и действия осложняющих факторов [14-16], по математической конструкции связей (корреляций) между ключевыми точками изначально распределенного подъемника остается упрощенной.

2. Редуцированный (упрощенный) характер описания с минимальным количеством настраиваемых параметров позволяет рассчитывать на создание корректных процедур параметрического оценивания, реализуемых по месту организации служб диспетчерского контроля и сбора первичных данных.

3. Идентификация в классе упрощенных моделей, в нашем случае - это, прежде всего, допущение о постоянстве удельного веса газо-жидкостной смеси (ГЖС) среднего по стволу подъёмника, по сути, означает аппроксимацию реальных наблюдаемых процессов классом заданных уравнений. Возможное снижение точности моделирования, сужающее горизонты прогноза и планирования, может компенсироваться регламентом регулярной подстройки параметров в обязательном порядке при смене режимных состояний системы.

4. Программе производственных испытаний предшествует этап анализа проблемных задач оценивания на модельных экспериментах с «зашумлёнными измерениями». Результаты данного этапа обсуждаются в заключительной части работы. Поверка работоспособности алгоритма по данным глубинного контроля осуществляется в части идентификации параметров притока по незавершённым [17] пуско-остановочным режимам работы скважины.

Функциональная схема скважины, приведенная к вертикали, изображенная на рис. 1, иллюстрирует принятые обозначения переменных состояний $(k-$ координата дискретного времени) и параметров:

- $\quad p_{R}, p(2, k), p(1, k), p(3, k), p(4, k), p(8, k), p_{L}(k)$ - распределение давлений [МПа] вдоль линии тока от пласта, призабойной зоны (ПЗ), забоя на уровне $H_{R}$, подвески насоса на уровне $H_{N}$, затрубья до перепускного клапана, выход насосно-компрессорной трубы (НКТ), до линейного нефтесборника;

- $q(k), q_{N}(k), q_{T}(k), q_{L}(k)$ - объемные расходы [ ${ }^{3} /$ сут] ГЖС с обводненностью $\beta$ и средним удельным весом $\gamma$ по стволу скважины [MПа/м] от забоя $q(k)=q_{N}(k)+q_{T}(k)$ по каналам НКТ, затрубья (3Т) и на устье;

- $r_{U}, r_{G U}, r_{T}, r_{N}, r_{K}$ - известные по данным конструкции гидросопротивления, линеаризованной модели потерь напора на трение на устьевом штуцере, перепускном клапане и в сегментах подъемника: затрубье, НКТ, низ обсадной колонны [сут/м²];

- кусочно-линейная нормированная напорная характеристика насоса задается таблицей своих коэффициентов $\left\langle\lambda_{0}(i), \lambda_{1}(i)\right\rangle$ (рис. 2 в [6]), в общем случае настраиваемых по каталогам изделий $[18,19]$, и параметрами типоразмера силовой установки $\left\langle h^{0}, q^{0}\right\rangle$ по номинальным значениям напора $[\mathrm{M}]$ и расхода $\left[\mathrm{m}^{3} / \mathrm{cyт}\right]$;

- переменные состояния по входу: $u(k)=\{1,0\}$ идентификатор режима работы по типу \{ВКЛ, ВЫКЛ $\}, \omega(k)$ - относительная частота вращения вала двигателя, в случае частотно-регулируемого привода пропорциональная частоте питающего напряжения (в номинале $\omega(k)=1)$.

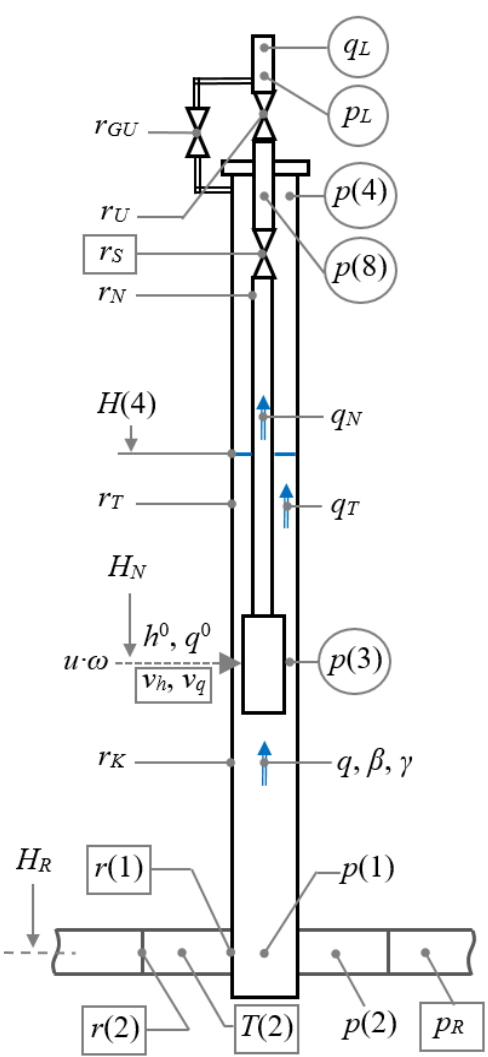

Pис. 1. Функциональная схема скважины с погружным электронасосом (отдельно выделены измеряемые переменные состояния и идентифичируемыле параметры)

Fig. 1. Functional diagram of a well with a submersible electric pump (measured state variables and identified parameters are separately highlighted)

Параметры модели, подлежащие оцениванию, делятся на две части.

Первая часть (дрейфующие характеристики подъемника):

- $r_{S}$ - дополнительное гидросопротивление НКТ, обусловленное сорбированием парафинов, солей и прочих поллютантов (изначально $r_{S}=0$ );

- $v_{h}, v_{q}$ - коэффициенты деформации (снижения) напорной характеристики насоса по напору (износ) и расходу (засорение) (изначальное $v_{h}=v_{q}=1$ ). Вторая часть (дрейфующие параметры притока):

- $p_{R}$ - давление подпора пласта на контуре питания $\Pi 3$;

- $r(2)$ - гидросопротивление перехода «пласт-ПЗ»; 
- $r(1)$ - гидросопротивление перехода «ПЗ-забой» или их обратные величины $w(2)=1 / r(2)$, $w(1)=1 / r(1)$, именуемые гидропроводностями переходов, которые в совокупности дают оценку коэффициенту продуктивности линейной модели притока скважины $w_{R}=\frac{w(1) w(2)}{w(1)+w(2)}$;

- $\quad T(2)$ - постоянная времени ПЗ, связанная с параметром ее объемной гидроупругости: $T(2)=r(2) \tau(2)$ [20], в первом приближении оценивается по длительности периода окончания переходного про-

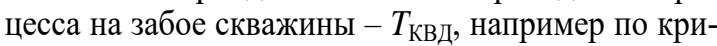
вой восстановления давления (КВД) [7, 14]

$$
T(2)=T_{\text {Квд }} / 3 \text {. }
$$

Обратим внимание на ряд особенностей организации работы процедур параметрического оценивания.

1. Выделенные на рис. 1 контролируемые переменные состояния имеют разномасштабные периоды измерений:

- состояния по давлениям, равно как и по регулируемой частоте

$I_{P}=\left\langle p_{L}(k), p(4, k), p(8, k), p(3, k), \omega(k) \quad k \in K\right\rangle,(2)$

считываемые в моменты времени $t_{k}$, имеют сравнительно высокую частоту опроса $t_{k+1}=t_{k}+\Delta t_{p}, \Delta t_{p} \sim 30 \mathrm{c}$, $k \in K=\{0,1,2, \ldots\}$ - идентификатор дискретного времени по шкале «плотных» измерений;

- замеры расходов на устье

$$
I_{q}=\left\langle q_{L}(k), \quad k \in K_{q} \subset K\right\rangle
$$

осуществляются намного реже $\Delta t_{q} \sim 30$ мин и более, $K_{q}$ - шкала «редких» измерений, синхронизированная по времени со шкалой $K$.

2. Контроль давления у приема насоса - $p(3, k)$ - позволяет разбить описание модели и, как следствие, алгоритм оценивания на два блока в соответствии с указанным ранее разделением идентифицируемых параметров. Реализуемая таким образом декомпозиция задачи идентификации шестимерного вектора на две задачи меньшей размерности с векторами

$$
\mathbf{c}_{N}=\left[\begin{array}{lll}
r_{1} & \delta v & v_{h}
\end{array}\right]^{T}, \mathbf{c}_{R}=\left[\begin{array}{lll}
p_{R} & r(1) & r(2)
\end{array}\right]^{T},
$$

где $r_{1}=r_{N}+r_{S}, \delta v=v_{h} / v_{q}$ повышает уровень обусловленности оценок по методу наименьших квадратов (MHK).

3. Условия идентифицируемости параметров (4) модели скважины напрямую зависят от уровня информативности (вариабельности) данных контроля (2), (3), что чаще не обеспечивается стационарными режимами нормальной эксплуатации системы. Типовые методики анализа притока по КВД [7, 20] не экономичны в технологиях реального времени, не информативны по состоянию подъемника и могут быть не корректными по нелинейным проявлениям, связанным с наличием свободного газа на забое. Должный уровень информативности данных первичного контроля обеспечивается в нашем случае режимом пробных частотных возмущений $\omega(k), k \in K$ работы ЭЦН (без останова скважины).

В отличие от классических методов анализа целых решений, например по КВД, где метод пробных возмущений для произвольных $\omega(k)$ не применим, в рассматриваемой схеме анализа переходных процессов метод пробных возмущений [21] оказывается не только возможным, но и необходимым инструментом повышения корректности решения обратной задачи.

Отмеченное выше разделение модели от состояния $p(3, k)$ на составные блоки сверху и снизу включает:

Канал «насос-HKT», описываемый в условиях $q_{L}(k)>0$ $[6,16]$ равенством

$$
\begin{gathered}
p(3, k)+\gamma\left[\begin{array}{l}
u(k) \omega(k)^{2} v_{h} h^{0} \lambda_{0}(i)- \\
-u(k) \omega(k) \frac{v_{h} h^{0}}{v_{q} q^{0}} \lambda_{1}(i) q_{N}(k)
\end{array}\right]= \\
=p(8, k)+\gamma\left(H_{N}+r_{1} q_{N}(k)\right),
\end{gathered}
$$

где $i$ - номер участка напорной характеристики (рис. 2 в [6]), определяемый условием

$$
\left.\left.i=\arg \left\{\frac{q_{N}(k)}{\omega(k) \cdot q^{0}} \in v_{q}\right] q N(i+1), q N(i)\right]\right\} .
$$

Возможный режим противотока в НКТ $\left(q_{N}(k)<0\right.$, $\left.H(8, k)>0,1_{q}(k)=1[6]\right)$ в данном описании игнорируется.

Канал «затрубье», описываемый равенством

$$
\begin{gathered}
p(3, k)=p(4, k)+ \\
+\gamma\left(H_{N}-H(4, k)-\left(1-1_{4}(k)\right) r_{T}\left(q(k)-q_{N}(k)\right)\right),
\end{gathered}
$$

где динамический уровень раздела фаз $H(4, k)$ с идентификатором отсутствия подачи через перепускной клапан из затрубья $\left(1_{4}(k)=1\right)$, с площадью кольцевого сечения $-S_{T}$ описывается уравнением

$$
S_{T} \frac{d}{d t} H(4, t)=1_{4}(t)\left(q_{N}(t)-q(t)\right) .
$$

Канал «низ колоньл»

$$
p(1, k)=p(3, k)+\gamma\left(H_{R}-H_{N}+r_{K} q(k)\right) .
$$

Блок притока

$$
\begin{gathered}
(T(2) D+1) p(2, k)=p_{R}-r(2) q(k), \\
D=d / d t, \\
q(k)=w(1) \frac{p(1, k)(p(2, k)-p(1, k))}{p(1, k)+\mu_{R}\left(p_{G O}-p(1, k)\right)_{+}}, \\
\mu_{R}=\frac{(1-\beta) G \cdot p_{0}}{p_{G O}-p_{0}},
\end{gathered}
$$

где $G$ - газовый фактор нефти [22], $p_{G O}$ - давление насыщения, ниже которого инициируются процессы разгазирования нефти, $p_{0} \approx 0,1 \mathrm{MПа} \mathrm{-} \mathrm{давление} \mathrm{атмо-}$ сферное.

Техника параметрического оценивания по МНК $[23,24]$, связанная с приведением модели объекта идентификации к регрессионному виду

$$
y(k)=\mathbf{c}^{T} \mathbf{v}(k),
$$


основана на построении и последующем решении системы линейных алгебраических уравнений

$$
\begin{gathered}
F \cdot \hat{\mathbf{c}}=\mathbf{b}, F=\sum_{k \in K_{O}} \mu(k) \mathbf{v}(k) \mathbf{v}(k)^{T}, \\
\mathbf{b}=\sum_{k \in K_{O}} \mu(k) \mathbf{v}(k) y(k)
\end{gathered}
$$

относительно вектора оценок искомых параметров. Здесь $\mu(k) \geq 0$ - весовые коэффициенты $\sum_{k \in K} \mu(k)=1$, ранжирующие значимость данных первичного контроля (2), (3) с использованием которых формируются вторичные данные $I_{U}=\left\langle y(k), \mathbf{v}(k) k \in K_{O} \subset K\right\rangle$, предназначенные для реализации алгоритма (10). В приме$\operatorname{pax} \mu(k)=1 / N_{0}$, где $N_{0}$ - мощность множества $K_{0}$ (число слагаемых в сумме, охватывающих значимую часть возмущённого режима работы системы). (9)

Преобразование модели (5) к регрессионному виду

$$
y_{N}(k)=\mathbf{c}_{N}{ }^{T} \mathbf{v}_{N}(k)
$$

устанавливает правило формирования пары

$$
\begin{aligned}
& y_{N}(k)=\frac{1}{\gamma}(p(3, k)-p(8, k))-H_{N} \\
& \mathbf{v}_{N}(k)= \\
& =\left[\begin{array}{lll}
q_{N}(k) & u(k) \omega(k) q_{N}(k) \frac{h^{0}}{q^{0}} \lambda_{1}(i) & -u(k) \omega(k)^{2} h^{0} \lambda_{0}(i)
\end{array}\right]^{T}
\end{aligned}
$$

и, согласно (10), алгоритма оценивания неизвестных (дрейфующих) параметров подъемника

$$
F_{N} \cdot \hat{\mathbf{c}}_{N}=\mathbf{b}_{N} .
$$

Реализация алгоритма возможна, если пара (11) доступна формированию по данным $I_{P}$ и $I_{q}$ первичного контроля (2), (3). Если выход регрессионной модели $y_{N}(k)(11)$ формируется, согласно (5), по «плотной» шкале $k \in K$ измерений $I_{P}$, то восстановление компонент вектора $v_{N}(k)$ связано с измерениями расходов $q_{N}(k)$. Заметим, что устьевые замеры расходов $q_{L}(k)$ осуществляются по «редкой» временной шкале $I_{q}$, $k \in K_{q}(3)$, причем равенство $q_{L}(k)=q_{N}(k)$ имеет место в условиях $1_{4}(k)=1$, когда подача $q_{T}(k)$ через перепускной клапан отсутствует (6).

Если одновременно с НКТ наблюдается подача через клапан $1_{4}(k)=0, \quad H(4, k)=0, \quad$ то выполнено $q_{L}(k)=q(k)$ и на основании (6) можно записать

$$
\begin{gathered}
q_{N}(k)=q_{L}(k)-\frac{1}{r_{T}}\left(1-1_{4}(k)\right) \cdot y_{T}(k), \\
y_{T}(k)=\frac{1}{\gamma}(p(3, k)-p(4, k))-H_{N},
\end{gathered}
$$

что свидетельствует о возможности формирования переменной $q_{N}(k)$ по шкале «редких» замеров $-K_{q}$ следовательно, и о реализуемости алгоритма (12).

Построение регрессионной модели для неизвестных параметров (4) притока (8) в виде

$$
y_{R}(k)=\mathbf{c}_{R}{ }^{T} \mathbf{v}_{R}(k)
$$

в условиях, что оценка $T(2)$ (1) априорно известна, связано со следующими преобразованиями уравнений (8).

Используя алгебру линейных дифференциальных операторов $[25,26]$, перепишем модель динамики П3 (8) в виде:

$$
p(2, k)=p_{R}-r(2) \tilde{q}(k),
$$

где $q(k)$ воспроизводится на выходе реализуемого динамического фильтра состояний [23]

$$
(T(2) D+1) \tilde{q}(k)=q(k) .
$$

Подставляя (15) в выражение для притока в забой скважины (8), после приведения к общему знаменателю и деления результата на $w(1) \cdot p(1, k)$, приходим к искомому представлению пары (14) в виде

$$
\begin{gathered}
y_{R}(k)=p(1, k), \\
\mathbf{v}_{R}(k)=\left[\begin{array}{lll}
1 & -\delta_{P}(1, k) q(k) & -\tilde{q}(k)
\end{array}\right]^{T},
\end{gathered}
$$

где

$$
\delta_{P}(1, k)=1+\mu_{R}\left(\frac{p_{G 0}}{p(1, k)}-1\right)_{+}
$$

и алгоритму оценивания по правилу (10)

$$
F_{R} \cdot \hat{\mathbf{c}}_{R}=\mathbf{b}_{R} .
$$

Как и в случае с (12), реализация алгоритма (18) возможна, если пара (17) доступна формированию по данным контроля (2), (3).

Согласно (17), формирование пары $\left\langle y_{R}(k), \mathbf{v}_{R}(k)\right\rangle$ основывается на расчетах давления - $p(1, k)$, переменной $q(k)$ и ее динамического аналога $-\tilde{q}(k)$, восстановленного с помощью фильтра (16). В отличие от схемы формирования данных (11) для идентификации параметров подъемника (12), которые, по сути, собираются по шкале «редких» измерений $-K_{q}$, корректное воспроизведение выхода фильтра на возмущенных режимах эксплуатации динамический системы требует построения пары (17) по более «плотной» временной шкале с периодами квантования $\Delta t_{0}$ менее $T_{2} / 30 \sim 10 \ldots 15$ мин, что в условиях формирования выборки $I_{q}(3)$ может быть недостижимым.

Рассмотрим схему построения оценок $\hat{q}(k), \hat{q}_{N}(k)$, $k \in K$ процессов $q(k), q_{N}(k)$ на основе реализации настраиваемого наблюдателя [27-30].

В условиях реализации первичной процедуры МНК оценивания параметров подъемника: $\hat{\mathbf{c}}_{\mathbf{N}}=\left[\begin{array}{lll}\hat{r}_{1} & \delta \hat{v} & \hat{v}_{h}\end{array}\right]^{T}$, динамика расхода в НКТ $-q_{N}(k)$, согласно (5) и (12), может быть оценена расчетным путем на основе данных $I_{P}$ с «плотной» временной шкалой по выражению

$$
q_{N}(k)=\left[\frac{u(k) \omega(k)^{2} \hat{v}_{h} h^{0} \lambda_{0}(i)+y_{N}(k)}{\hat{r}_{1}+u(k) \omega(k) \delta v \frac{h^{0}}{q^{0}} \lambda_{1}(i)}\right]_{+}, k \in K,
$$

где учтена блокировка возможного противотока $q_{N}(k)<0$ герметичным обратным клапаном на выкиде 
ЭЦН (знак «+» на позиции индекса) при выключении $(u(k) \rightarrow 0)$ насоса.

В режиме $1_{4}(k)=0$, согласно $(13)$, искомая оценка притока $q(k)$ рассчитывается с использованием $\hat{q}_{N}(k)$ по правилу

$$
q(k)=q_{N}(k)+\frac{1}{r_{T}}\left(1-1_{4}(k)\right) \cdot y_{T}(k) .
$$

Но в условиях $1_{4}(k)=1$, когда динамика движения уровня $H(4, k)$, равно как и расхода $q_{T}(k)$, остаётся скрытой, приведённое соотношение оказывается неполным для оценивания притока $q(k)$. Для решения возникшей задачи воспользуемся моделью «затрубъя» (6), из которой, согласно (13), в условиях $1_{4}(k)=1$, следует

$$
\begin{gathered}
q(k)=q_{N}(k)-S_{T} \dot{H}(4, k), \\
H(4, k)=H_{N}-\frac{1}{\gamma}(p(3, k)-p(4, k))=-y_{T}(k) .
\end{gathered}
$$

Отсюда, наблюдатель притока для любых состояний $1_{4}(k)$ принимает вид

$$
q(k)=q_{N}(k)+1_{4}(k) S_{T} \dot{y}_{T}(k)+\frac{1}{r_{T}}\left(1-1_{4}(k)\right) \cdot y_{T}(k) .
$$

Построение оценки переменной $\hat{p}(1, k)$ по «плотной» временной шкале $k \in K$ осуществляется согласно (7) по выражению

$$
\hat{p}(1, k)=p(3, k)+\gamma\left(H_{R}-H_{N}+r_{K} \hat{q}(k)\right),
$$

где также используется ранее восстановленные (20) значения $\hat{q}(k)$.

Полученный ранее алгоритм идентификации параметров притока (17), (18) реконструируется с использованием оценок переменных $\langle\hat{p}(1, k), \quad \hat{q}(k), \quad \bar{q}(k)\rangle(20),(21)$ к виду

$$
\hat{F}_{R} \cdot \hat{\mathbf{c}}_{R}=\hat{\mathbf{b}}_{R}
$$

где вместо (16) переменная $\bar{q}(k)$ воспроизводится по правилу

$$
(T(2) D+1) \bar{q}(k)=\hat{q}(k)
$$

с «плотной» временной шкалой $k \in K_{q}$.

Вышеизложенное является доказательством следующего результата.

\section{Утверждение.}

Пусть гидродинамическая модель скважины с частотно-регулируемым ЭЦН (рис. 1), заданная системой уравнений «верха» (5), (6) с неопределенным вектором дрейфующих параметров $\mathbf{c}_{N} u$ «низа» (7), (8) с неопределенными параметрами притока $\mathbf{c}_{R}$ (4), представлена данными контроля $I_{P}$ и $I_{q}(2)$, (3), с разномасштабными интервалами («плотностью») синхронизированных измерений свочх состояний.

Тогда двухэтапная проиедура идентификаичи неизвестных параметров $\mathbf{c}_{N}, \mathbf{c}_{R}$ модели по данным контроля состояний $I_{P}$ и $I_{q}$, а также режима $1_{4}(k)$ подачи из затрубъя на основе МНК с одновременным очениванием динамики притока $\langle p(1, k), q(k)\rangle$, подачи $q_{N}(k)$ и уровня $H(4, k)=-y_{T}(k)$ посредством реализации настраиваемого по оченкам $\hat{\mathbf{c}}_{N}$ наблюдателя (19)-(23) с применением режима пробных частотных возмущений $\omega(k)$ ЭЦН задается нижеследующей инструкичей:

НАЧАЛО

1. Ввод исходных данных о параметрах конструкции, ЭЦН и флюида.

2. Синхронизачия данных выбборки $I_{P}$ и $I_{q}$ c вblсокой (2) $k \in K$ и низкой (3) $k \in K_{q}$ частотами onpoca.

3. Формирование данных входо-выходныхх состояний (11), (12) модели регрессии подъёмника по шкале $k \in K_{q}$.

4. Расчёт МНК оченки (12) параметров подъ$\ddot{е м м н и к а ~} \hat{\mathbf{c}}_{N}$.

5. Реализащии настраиваемого по $\hat{\mathbf{c}}_{N}$ наблюдателя (19)-(21), (23), с последующей расчётной оченкой графиков динамики притока $\langle p(1, k), \quad q(k)\rangle$ и входо-выходных состояний его модели регрессии (17) по шкале $k \in K$.

6. Расчёт МНК оченки (22) параметров притока $\hat{\mathbf{c}}_{R}$.

КОНЕЦ.

Тестирование представленного алгоритма идентификации осуществлялось по данным моделирования частотно $\omega(k)$ возмущённых режимов работы системы с настройками из табл. 1. Данные измерений формировались на основе модельных состояний с добавлением искусственной помехи измерений.

Результаты тестирования свидетельствуют, что по показателю относительной ошибки оценивания: $\delta \mathbf{c}_{i}=\left(\left|c_{i}-\hat{c}_{i}\right| / c_{i}\right) \cdot 100 \%$ реализуемый по модельным измерениям алгоритм идентификации доставляет практически точные оценки искомых параметров. Наблюдаемая малая погрешность оценки притока обусловлена ошибкой разностного вычисления производной сигнала $q_{T}(k)(20)$. Однако наложение помехи измерений (стандартная функция «rand» ПО MatLab [31]) с нулевым средним и амплитудами: 1 МПа для $p(3, k), 10 \mathrm{~m}^{3} /$ сут для $q_{L}(k)$ и $0,25 \mathrm{MПа} \mathrm{для}$ $p(4, k)$ и $p(4, k)$, уже искажает результат оценивания, что свидетельствует о плохой обусловленности рассматриваемых задач $\left(\operatorname{cond}\left(F_{\bullet}\right) \sim 10^{6}\right)$ и указывает как минимум на два момента:

1) необходимость предварительной фильтрации измеряемых сигналов;

2) необходимость применения процедур регуляризации алгоритмов МНК с внесением дополнительной априорной информации об искомых моделях.

Учитывая необходимость расчёта производной (20), решение первой задачи осуществлялось посредством аппроксимации зашумлённых сигналов контроля [32-34] на интервалах постоянства частотных возмущений гладкими кривыми, предоставляемыми инструментальной средой MatLab, модуль Curve Fitting Toolbox [31]. Сравнительный анализ результатов фильтрации позволил выбрать вариант базиса в виде дробно рациональный функции. 
Таблица 1. Параметры модели скважины

Table 1. Well model parameters

\begin{tabular}{|c|c|c|}
\hline Наименование, [ед. изм.] & Значение & Обозначение \\
\hline Name, [units] & Value & Notation \\
\hline $\begin{array}{l}\text { Среднепластовое давление, [MПа] } \\
\text { Average reservoir pressure, [MPa] }\end{array}$ & 22 & $p_{R}$ \\
\hline $\begin{array}{l}\text { Глубина забоя по вертикали, [м] } \\
\text { Well depth, [m] }\end{array}$ & 3000 & $H_{R}$ \\
\hline $\begin{array}{l}\text { Коэффициент продуктивности сква- } \\
\text { жины, [м³/(МПа·сут)] } \\
\text { Well productivity, }\left[\mathrm{m}^{3} /(\mathrm{MPa} \cdot \text { day)] }\right.\end{array}$ & 8 & $w_{R}$ \\
\hline $\begin{array}{l}\text { Коэффициент гидропроводности } \\
\text { притока из пласта в ПЗ скважины, } \\
\text { [м³/(МПа·сут)] } \\
\text { Reservoir productivity coefficient, } \\
{\left[\mathrm{m}^{3} /(\text { MPa·day)] }\right.}\end{array}$ & 10 & $w(1)$ \\
\hline 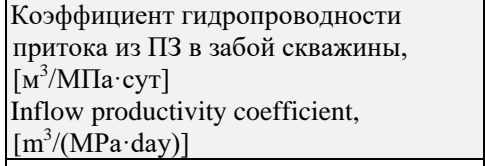 & 40 & $w(2)$ \\
\hline $\begin{array}{l}\text { Объёмная доля воды, [ед.] } \\
\text { Water cut, [units] }\end{array}$ & 0,3 & $\beta$ \\
\hline \multicolumn{3}{|l|}{$\begin{array}{l}\text { Удельный вес, }[\mathrm{MПа} / \mathrm{M}] \\
\text { Specific gravity, }[\mathrm{MPa} / \mathrm{m}]\end{array}$} \\
\hline •нефти/oil & $0,68 \cdot 10^{-2}$ & $\gamma_{0}$ \\
\hline •воды/water & $1,1 \cdot 10^{-2}$ & $\gamma_{W}$ \\
\hline •жидкости/liquid & $0,8 \cdot 10^{-2}$ & $\gamma$ \\
\hline •газа/gas & $0,8 \cdot 10^{-5}$ & $\gamma_{G 0}$ \\
\hline $\begin{array}{l}\text { Весовая характеристика газа, [MПа/м] } \\
\text { Gas weight characteristic, }[\mathrm{MPa} / \mathrm{m}]\end{array}$ & $5,6 \cdot 10^{-4}$ & $Y_{G}$ \\
\hline $\begin{array}{l}\text { Газовый фактор, [ед.] } \\
\text { Gas factor, [units] }\end{array}$ & 100 & $G$ \\
\hline \begin{tabular}{|l} 
Давление в линии нефтесбора, [МПа] \\
Oil collector line pressure, [MPa] \\
\end{tabular} & 1,5 & $p_{L}$ \\
\hline \begin{tabular}{|l|} 
Давление насыщения, [MПа] \\
Saturation pressure, [MPa] \\
\end{tabular} & 18 & $p_{G 0}$ \\
\hline \multicolumn{3}{|l|}{$\begin{array}{l}\left.\text { Гидросопротивления, [cyт/ } / \mathrm{m}^{2}\right] \\
\text { Hydroresistances, }\left[\mathrm{day} / \mathrm{m}^{2}\right]\end{array}$} \\
\hline • обсадной колоны/casing & 1 & $r_{K}$ \\
\hline •HKT/tubing & 1,6 & $r_{N}$ \\
\hline •устьевого штуцера/wellhead choke & 1 & $r_{U}$ \\
\hline •затрубного пространства/annulus & 0,8 & $r_{Z T}$ \\
\hline $\begin{array}{l}\text { • перепускного клапана из ЗТ } \\
\text { wellhead annulus choke }\end{array}$ & 2 & $r_{G U}$ \\
\hline $\begin{array}{r}\bullet \text { потерь на засорение в НКТ } \\
\text { pressure loss due to clogging }\end{array}$ & 1 & $r_{S}$ \\
\hline \multicolumn{3}{|l|}{$\begin{array}{l}\text { Параметры насоса } \\
\text { Pump parameters }\end{array}$} \\
\hline •уровень подвески, [м]/pump depth, [m] & 2200 & $H_{N}$ \\
\hline $\begin{array}{l}\bullet \text { номинальный напор, [м] } \\
\text { nominal head, [m] }\end{array}$ & 1900 & $h^{0}$ \\
\hline 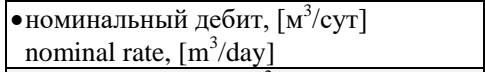 & 60 & $q^{0}$ \\
\hline \multicolumn{3}{|l|}{$\begin{array}{l}\text { Площади сечений, }\left[\mathrm{m}^{2}\right] \\
\text { cross-sectional areas, }\left[\mathrm{m}^{2}\right]\end{array}$} \\
\hline •HKT/tubbing & 0,002 & $S_{N}$ \\
\hline •3T/annulus & 0,01 & $S_{T}$ \\
\hline $\begin{array}{l}\text { Постоянная времени ПЗ, [сут] } \\
\text { Inflow zone response time, [day] }\end{array}$ & 0,4 & $T(2)$ \\
\hline
\end{tabular}

По аналогии с типовыми подходами ГдИС [7, 14] реконструкция (регуляризация) процедур оценивания (12), (22), сформированных по данным частотно возмущенных режимов работы, осуществлялась введением дополнительного уравнения связи, восстановленного для осреднённых состояний стационарных режимов эксплуатации до периода пробных возмущений. Правило реконструкции с понижением размерности матрицы МНК в следующем. Пусть исход- ная регрессионная модель объекта идентификации (9) имеет вид

$$
y(k)=c_{1} v_{1}(k)+c_{2} v_{2}(k)+c_{3} v_{3}(k), k \in K_{1} \bigcup K_{2},
$$

где $K_{1}=\left\{1,2, \ldots N_{1}\right\}$ - дискретные моменты времени стационарного режима работы, а $K_{2}=\left\{N_{1}+1, N_{1}+2, \ldots N_{2}\right\}-$ последующий период возмущений. В условиях зашумлённости данных измерений равновесное состояние системы на периоде $k \in K_{1}$ может быть представлено моделью

$$
\bar{y}=c_{1} \bar{v}_{1}+c_{2} \bar{v}_{2}+c_{3} \bar{v}_{3}
$$

с вычисляемыми осреднёнными состояниями

$$
\bar{y}=\sum_{k \in K_{1}} y(k) / N_{1}, \quad \bar{v}_{i}=\sum_{k \in K_{1}} v_{i}(k) / N_{1}, \quad i \in\{1,2,3\} .
$$

В условиях $v_{1}(k)>0, k \in K_{1} \cup K_{2}$ справедливо преобразование с переходом от (24) к модели и алгоритму меньшей размерности вида

$$
\begin{gathered}
y_{Z}(k)=\mathbf{c}_{Z}^{T} \mathbf{z}(k), F_{Z} \cdot \hat{\mathbf{c}}_{Z}=\mathbf{b}_{Z}, \\
\hat{c}_{1}=\left(\bar{y}-\hat{c}_{2} \bar{v}_{2}+\hat{c}_{3} \bar{v}_{3}\right) / \bar{v}_{1}, \hat{\mathbf{c}}_{Z}=\left[\hat{c}_{2} \hat{c}_{3}\right]^{T},
\end{gathered}
$$

где

$$
\begin{gathered}
F_{Z}=\sum_{k \in K_{2}} \mu(k) \mathbf{z}(k) \mathbf{z}(k)^{T}, \\
\mathbf{b}_{Z}=\sum_{k \in K_{2}} \mu(k) \mathbf{z}(k) y_{Z}(k), \mu(k)=1 / N_{2}-N_{1},
\end{gathered}
$$

а выход и регрессоры укороченной модели удовлетворяют условиям

$$
y_{Z}(k)=\frac{y(k)}{v_{1}(k)}-\frac{\bar{y}}{\bar{v}_{1}}, \mathbf{z}(k)=\left[\frac{v_{2}(k)}{v_{1}(k)}-\frac{\bar{v}_{2}}{\bar{v}_{1}} \frac{v_{3}(k)}{v_{1}(k)}-\frac{\bar{v}_{3}}{\bar{v}_{1}}\right]^{T} .
$$

Именно данная схема редукции была использования для реконструкции процедур оценивания подъёмника (12) и притока (22).

На рис. 2 вынесены типовые графики стационарного и последующих частотно возмущенных режимов работы системы с наложенной помехой измерений для ключевых состояний $p(3, k)$ - давления у приёма и $q_{L}(k)$ - объёмного расхода на устье. Отдельно на рис. 2 вынесены укрупнённые фрагменты исходных графиков, дополненные для сравнения кривыми результатов выходов наблюдателя, настроенного по результатам идентификации.

В серии из двадцати статистических реализаций наложенных помех измерений отдельно для трёх вариантов реализаций гидродинамики фильтра (23) с параметрами $T(2)=\{0,3 ; 0,4 ; 0,5\}$ (истинное значение $T(2)=0,4)$ сигнальная ошибка наблюдения на возмущённых режимах работы модели в среднем по всем экспериментам и сигналам не превышала $2 \%$. Осредненные данные результатов работы алгоритмов оценивания параметров подъёмника и притока сведены в табл. 2 и иллюстрируются диаграммами на рис. 3 .

Сравнительный анализ результатов параметрического оценивания свидетельствует, что относительная ошибка идентификации параметров деформации напорной характеристики насоса $\delta v$ и $v_{h}$ в среднем не превышает 4 и $6 \%$, в то время как ошибка идентифи- 
кации суммарного гидросопротивления подъёмника $r_{1}$ может доходить до 100 \%. При этом точность воспроизведения сигнальных процессов (рис. 2) на выходе наблюдателя не выходит за уровень в 2 \%. Подобная неравномерность ошибки оценивания имеет место и для параметров притока. Так, для давления пласта $-p_{R}$ и гидросопротивления $-r(1)$ ближней зоны притока ошибки идентификации во всех модельных экспериментах не превышают 2 и $5 \%$. В то время как ошибка по $r(2)$ доходит до $30 \%$, что, опятьтаки, не нарушает хорошую сходимость воспроизведения гидродинамики притока.

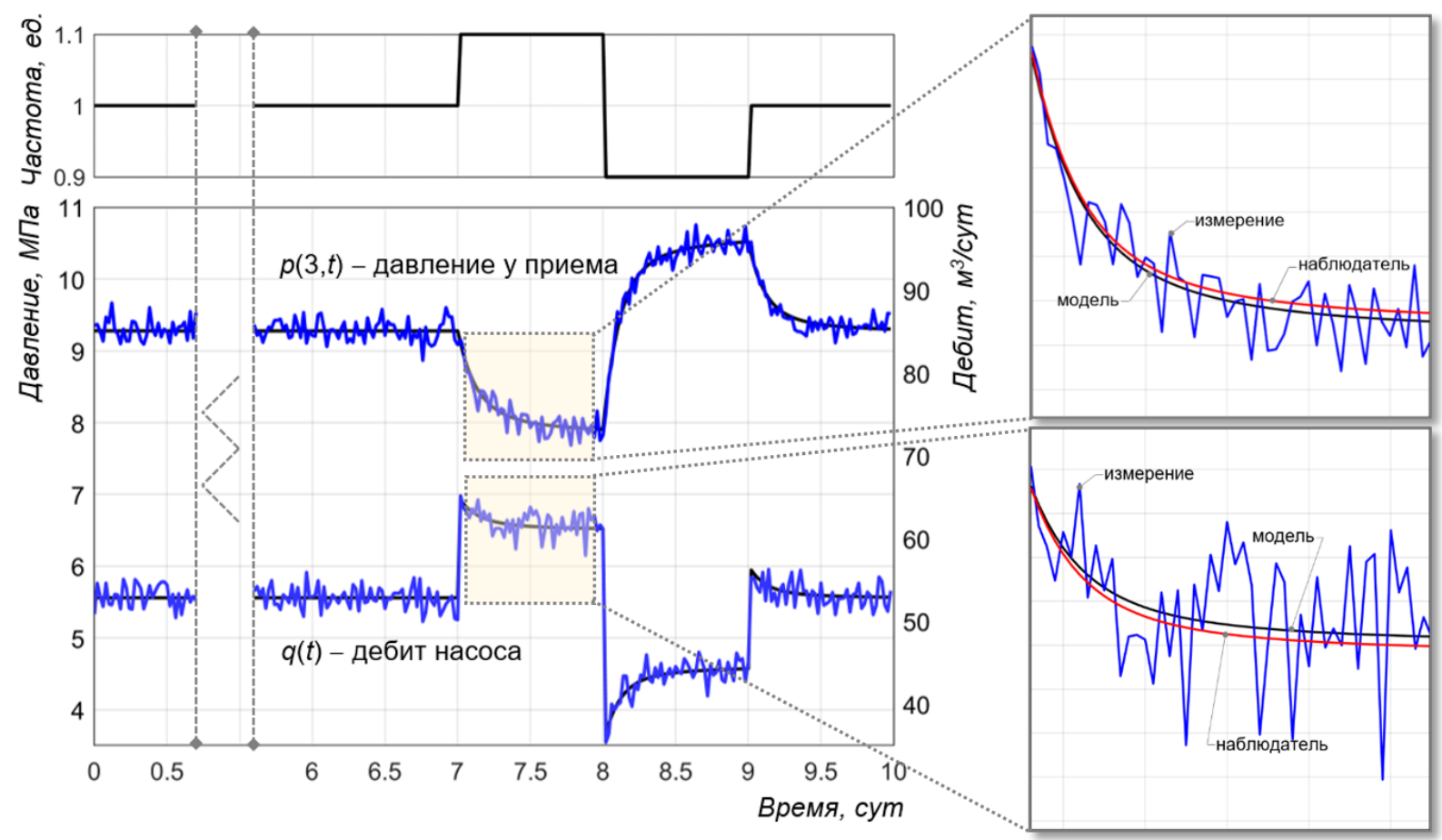

Pис. 2. Графики возмущённых переменных состояний моделирования скважины и тестовые «измерения» после наложения помехи максимальной амплитуды

Fig. 2. Well model perturbed variable states and test «measurements» with maximum amplitude noise

Таблица 2. Статистика результатов работы алгоритма идентификации параметров скважины по данным модельных экспериментов

Table 2. Statistics of the well parameters identification algorithm results on model experiments data

\begin{tabular}{|c|c|c|c|c|c|c|c|c|c|c|c|c|}
\hline \multirow{3}{*}{$\begin{array}{l}\text { Описание эксперимента } \\
\text { Experiment definition }\end{array}$} & \multicolumn{6}{|c|}{$\begin{array}{c}\text { Схема идентификации подъемника } \\
\text { Lift identification scheme }\end{array}$} & \multicolumn{6}{|c|}{$\begin{array}{l}\text { Схема идентификации притока } \\
\text { Inflow identification scheme }\end{array}$} \\
\hline & \multicolumn{3}{|c|}{$\begin{array}{l}\text { Параметр } \\
\text { Parameter }\end{array}$} & \multicolumn{3}{|c|}{$\begin{array}{c}\text { Ошибка оценива- } \\
\text { ния, \% } \\
\text { Estimation error } \\
\end{array}$} & \multicolumn{3}{|c|}{$\begin{array}{l}\text { Параметр } \\
\text { Parameter }\end{array}$} & \multicolumn{3}{|c|}{$\begin{array}{l}\text { Ошибка оценива- } \\
\text { ния, \% } \\
\text { Estimation error }\end{array}$} \\
\hline & $r_{1}$ & $\delta v$ & $v_{h}$ & $r_{1}$ & $\delta v$ & $v_{h}$ & $p_{r}$ & $r(1)$ & $r(2)$ & $p_{r}$ & $r(1)$ & $r(2)$ \\
\hline $\begin{array}{l}\text { Исходные параметры } \\
\text { Initial parameters }\end{array}$ & 2,84 & 1,125 & 0,9 & - & - & - & 22 & 0,1 & $0,025 \mid$ & - & - & - \\
\hline $\begin{array}{l}\text { Идентификация без помехи и фильтра } \\
\text { Identification without noise and filter }\end{array}$ & 2,839 & 1,125 & 0,899 & 0,002 & 0,005 & 0,001 & 21,96 & 0,098 & 0,025 & 0,15 & 1,2 & 2,05 \\
\hline $\begin{array}{l}\text { Идентификация без помехи с фильтром } \\
\text { Identification without noise and with filter }\end{array}$ & 2,45 & 1,121 & 0,892 & 13,7 & 0,4 & 0,82 & 22,96 & 0,107 & 0,023 & 0,17 & 1,3 & 5 \\
\hline $\begin{array}{l}\text { Идентификация с помехой и фильтром } \\
\text { (среднее значение при } T(2)=0,3 \text { ) } \\
\text { Identification with noise and filter (average for } T(2)=0,3 \text { ) }\end{array}$ & 1,28 & 1,11 & 0,87 & 76,1 & 3,5 & 4,9 & $21,87 \mid$ & 0,10 & 0,03 & 0,7 & 3,0 & 16,3 \\
\hline $\begin{array}{l}\text { Идентификация с помехой и фильтром } \\
\text { (среднее значение при } T(2)=0,4 \text { ) } \\
\text { Identification with noise and filter (average for } T(2)=0,4 \text { ) }\end{array}$ & 0,93 & 1,10 & 0,86 & 80,3 & 2,8 & 4,4 & 21,99 & 0,10 & 0,03 & 0,8 & 2,4 & 17,7 \\
\hline $\begin{array}{l}\text { Идентификация с помехой и фильтром } \\
\text { (среднее значение при } T(2)=0,5 \text { ) } \\
\text { Identification with noise and filter (average for } T(2)=0,5 \text { ) }\end{array}$ & 1,39 & 1,11 & 0,87 & 99,7 & 4,0 & 5,1 & 22,12 & 0,10 & 0,02 & 1,0 & 3,5 & 16,8 \\
\hline
\end{tabular}



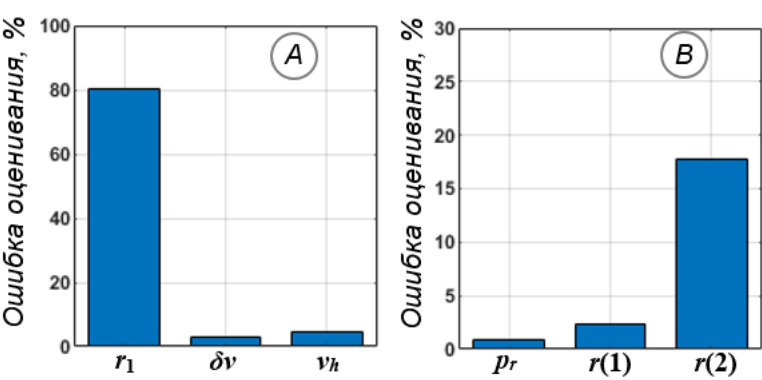

Pис. 3. Диаграммы средних ошибок оченивания параметров подъемника (A) и притока (B)

Fig. 3. Diagrams of average errors in estimating elevator (A) and inflow (B) model parameters
Для проверки работы системы по натурным измерениям использовалась выгрузка данных глубинного контроля давления у приёма со станции управления ЭЦН на незавершённом пуско-остановочном режиме работы скважины одного из месторождений Ноябрьского региона. Исходная информация по скважине и устьевым измерениям сведена в табл. 3 и представлена графиками на рис. 4. Нормированная напорная характеристика насоса, используемая при расчете наблюдателя в схеме идентификации притока, соответствует типовой из [6].

Таблица 3. Параметры модели, результаты ГДИС и идентификаџии

Table 3. Model parameters, test and identification results

\begin{tabular}{|c|c|c|c|c|c|c|c|c|c|c|}
\hline & \multicolumn{6}{|c|}{ Конструкция скважины/Well structure } & \multicolumn{4}{|c|}{ Параметры флюида/Fluid parameters } \\
\hline Параметр/Parameter & $H_{R}$ & $S_{N}$ & $S_{T}$ & $H_{N}$ & $h^{0}$ & $q^{0}$ & $\gamma_{O}$ & $\gamma_{W}$ & $\beta$ & $G$ \\
\hline Единицы измерения/Units & $\begin{array}{l}\mathrm{M} \\
\mathrm{m} \\
\end{array}$ & \multicolumn{2}{|c|}{$\begin{array}{l}\mathrm{M}^{2} \\
\mathrm{~m}^{2}\end{array}$} & \multicolumn{2}{|c|}{$\begin{array}{l}\mathrm{M} \\
\mathrm{m}\end{array}$} & $\begin{array}{l}\mathrm{M}^{3} / \mathrm{cyT} \\
\mathrm{m}^{3} / \text { day }\end{array}$ & \multicolumn{2}{|c|}{$\begin{array}{l}\mathrm{M} / \mathrm{M} / \mathrm{M} \\
\mathrm{MPa} / \mathrm{m}\end{array}$} & \multicolumn{2}{|c|}{$\begin{array}{l}\text { ед. } \\
\text { units }\end{array}$} \\
\hline Значение/Value & 2102 & 0,0042 & 0,056 & 1569 & 1670 & 200 & $0,81 \cdot 10^{-2}$ & $1,09 \cdot 10^{-2}$ & 0,07 & 13 \\
\hline & \multicolumn{3}{|c|}{$\begin{array}{l}\text { Результаты ГДИС } \\
\text { Inflow test results } \\
\end{array}$} & \multicolumn{3}{|c|}{$\begin{array}{c}\text { Расчетные параметры } \\
\text { Calculated parapeters }\end{array}$} & \multicolumn{4}{|c|}{$\begin{array}{c}\text { Результаты идентификации } \\
\text { Identification results } \\
\end{array}$} \\
\hline Параметр/Parameter & $\overline{p_{R}}$ & $\bar{w}_{1}$ & & $T(2)$ & & $r_{1}$ & $\overline{p_{R}}$ & $w(1)$ & $w(2)$ & $\overline{w_{R}}$ \\
\hline Единицы измерения/Units & $\begin{array}{l}\mathrm{M} \Pi \mathrm{a} \\
\mathrm{MPa}\end{array}$ & $\begin{array}{l}\mathrm{M}^{3} /(\mathrm{M \Pi} \\
\mathrm{m}^{3} /(\mathrm{MP}\end{array}$ & & $\begin{array}{l}\text { cyt } \\
\text { day }\end{array}$ & & $\begin{array}{l}\text { cyT } / M^{2} \\
\text { day } / m^{2}\end{array}$ & $\begin{array}{l}\mathrm{M \Pi а} \\
\mathrm{MPa}\end{array}$ & & 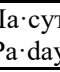 & \\
\hline Значение/Value & 13,3 & 31 & & 2 & & 1 & 13,09 & 38,5 & 108 & 28,4 \\
\hline
\end{tabular}

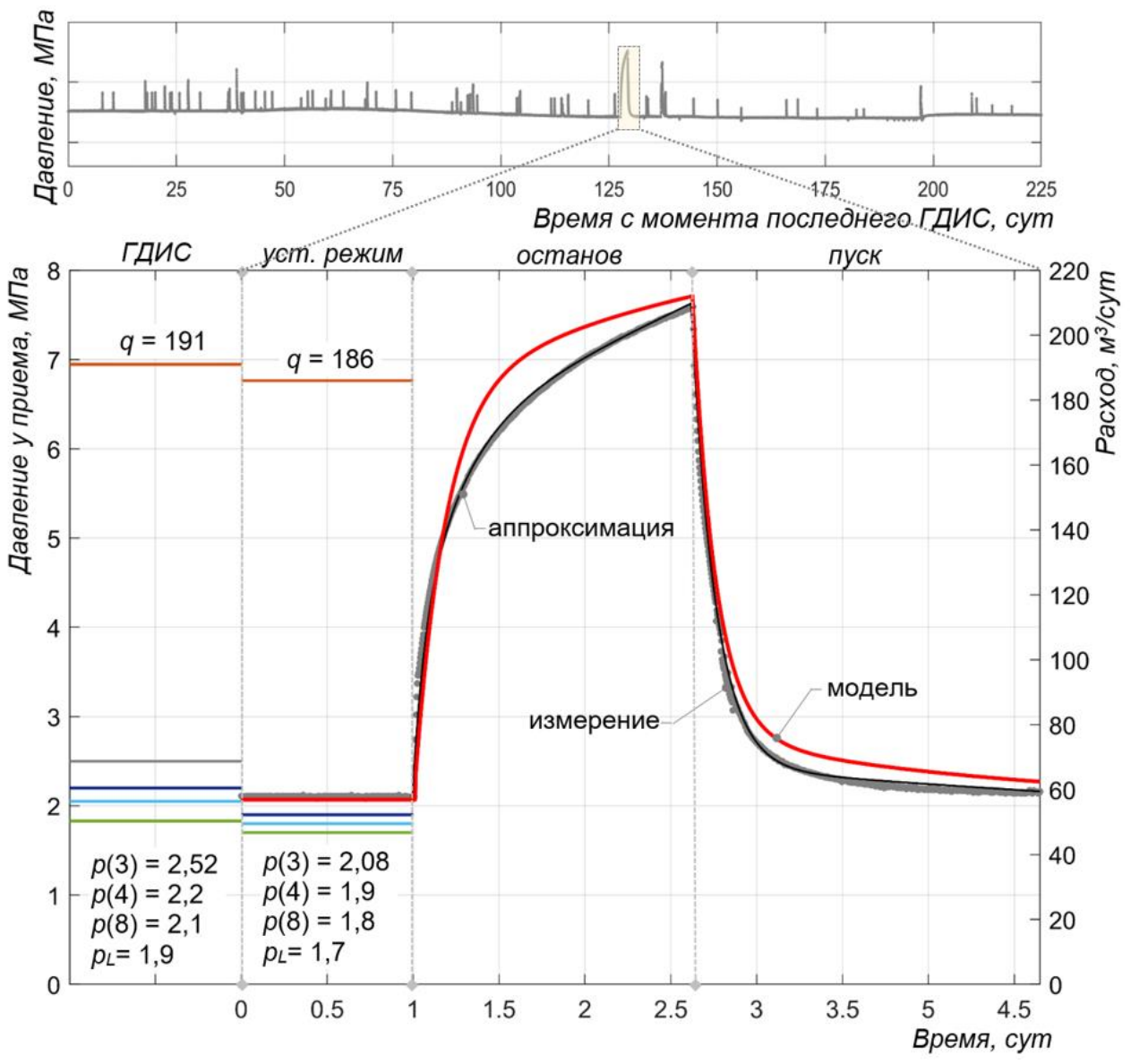

Рис. 4. Графики измерения давлений у приёма насоса при останове и повторном запуске, их аппроксимации и результаты моделирования, а также среднесуточные данные о равновесных устьевых давлениях и расходах перед ГДИС и анализируемым переходным прочессом

Fig. 4. Pump intake pressure charts during stop and consecutive start, their approximations and model results and daily average data of steady intake pressures and rates before well test and analyzed transient process 
Как видно из графика, шумы измерений незначительны, поэтому аппроксимация графика точечных измерений давления у приёма насоса непрерывной функцией использовалась для аналитического расчёта производной сигнала $p(3, k)$ для оценки $q(k)(20)$, с постоянным среднесуточным уровнем давления $p(4)$. Результат МНК идентификации по правилу (19)-(23) указывают, что ошибка параметрического оценивания относительно данных ГДИС (пластовое давление и коэффициент продуктивности) лежит в пределах 5 \%, а сигнальная ошибка по графику $p(3, k)$ не превышает $6 \%$, что приемлемо для задач диспетчерского контроля режимных условий эксплуатации системы.

\section{Выводы}

1. Рассмотренный регламент идентификации, ориентированный на задачи управления процессами эксплуатации скважин с ЭЦН, позволяет на регулярной основе отслеживать возможные дрейфы параметров подъёмника и притока, классифицируя тем самым первопричины нарушений режимных состояний работы оборудования и плановых показателей добычи.

2. Корректная работа алгоритма в условиях непрерывной эксплуатации скважины во многом обеспечивается следующими обстоятельствами:

- наличием точки глубинного контроля давления $p(3, k)$ у приёма насоса, позволяющей декомпозировать комплексную задачу идентификации с плохо различимыми вариациями контролируемых устьевых состояний на две задачи меньшей размерности с информативным сигналом $p(3, k)$ и более высокими факторами надёжности МНК оценок;

- введением процедуры пробных частотных возмущений, обеспечивающих повышенный уровень информативности данных контроля состояний на переходных режимах, без останова ЭЦН. Точечный контроль на интервалах пробных возмущений позволяет отслеживать дрейфы параметров модели с заданной периодичностью или по запросу оператора, что более практично в задачах реального времени, чем в классических пуско-остановочных регламентах контроля [35];

- предварительной фильтрацией зашумленных данных первичного контроля путём аппроксимации их гладкими кривыми, что позволяет корректно восстанавливать исходные сигналы и их производные, сокращая уровни относительных ошибок оценивания;

\section{СПИСОК ЛИТЕРАТУРЫ}

1. Proactive utilization of ESP performance monitoring to enhance productivity / Hashim Al-Sadah, Mohannad Abo Khamseen, Abdullah Al-Ghamdi, Ali Fardan // SPE middle east oil and gas show and conference. - Bahrain, 2019. URL: https://doi.org/10.2118/194925-MS

2. Rapid and comprehensive artificial lift systems performance analysis through data analytics, diagnostics and solution evaluation / Lichi Deng, Ehsan Davani, Hamed Darabi, Vural Sander Suicmez, David Castineira // SPE middle east artificial conference and
- приемами регуляризации на основе осреднения данных стационарных состояний регрессоров, восстановленных по равновесному состоянию системы до периода пробных возмущений.

3. Проведенные исследования, выявленные проблемные точки конструирования и корректного оценивания свидетельствуют, что рассмотренный вариант решения задачи идентификации, наблюдаемая неравномерная точность оценивания параметров подъёмника и притока никак не закрывают проблему автоматического сопровождения модели скважин с ЭЦН в условиях реальной эксплуатации. Однако требование к точности идентификации параметра $r_{1}$ подъёмника следует соотносить с условиями инициализации мероприятий по очистке НКТ от сорбентов, когда наблюдаемая производительность системы $q_{L}(k)$ заметно снижается на десять и более процентов. Причины таких последствий система идентификации опознаёт более надёжно.

Типовые правила ГДИС оценивают два главных показателя: подпор пласта на контуре питания $-p_{R}$ и суммарное гидросопротивление притока $r_{R}=r(1)+r(2)$, связанное с коэффициентом продуктивности скважины условием $w_{R}=1 / r_{R}$. Согласно статистике машинных испытаний (табл. 2), средняя относительная ошибка оценивания параметра $r_{R}$ в нашем случае не превышает $4 \%$.

\section{Выводы по результатам натурных измерений}

1. Динамика давлений для скважины с ЭЦН заметно отличается на пуске и останове, что связано с наличием в системе нелинейного звена - насоса с особым режимом импульсной откачки из затрубья в момент пуска.

2. Рассмотренная схема оценивания учитывает данную особенность и позволяет решать задачу идентификации притока в условиях пусков и остановов и при скачкообразных возмущениях частоты.

3. Предпринятая аппроксимация динамики призабойной зоны моделью первого порядка оказывается грубой для «хорошего» воспроизведения реальных переходных режимов притока, что на незавершённых процессах [17] может приводить к смещению оценок пары $\left\langle p_{R}, w_{R}\right\rangle$. Относительно данных ГДИС ошибка оценивания по указанным параметрам в приведённом анализе лежит в пределах $5 \%$, что приемлемо для задач оперативного контроля причин отклонений технологического режима от плана, но критично для задач с горизонтами длительного прогнозирования.

exhibition, - Bahrain, 2018. URL: https://doi.org/10.2118/192460MS (дата обращения 10.12.2019).

3. Mriganga Das, Zadil Hayat Bin Naharuddin, Dhuha Al-Khalid. Optimizing production and operation through automated well and network modeling // SPE Kuwait oil and gas show and conference. - Kuwait, 2015. URL: https://doi.org/10.2118/175205-MS (дата обращения 10.12.2019).

4. Reda El Mahbes, Wilfried Manfoumbi, Benedicte Kadio-Morokro. Real-time remote monitoring to enhance electrical submersible 
pump system run life and maximize production // SPE middle east artificial lift conference and exhibition. - Bahrain, 2018. URL: https://doi.org/10.2118/192470-MS (дата обращения 10.12.2019)

5. Gabor Takacs. Three inventions shaping the future of ESP technology // Journal of Petroleum Science and Engineering. 2019. - V. 182. URL: https://doi.org/10.1016/j.petrol.2019.106330 (дата обращения 10.12.2019).

6. Модель переходных процессов в скважине с частотнорегулируемым электроцентробежным насосом / И.Г. Соловьёв, Д.А. Говорков, С.С. Белашевский, Ю.А. Ведерникова // Известия Томского политехнического университета. Инжиниринг георесурсов. - 2019. - T. 330. - № 1. - C. 110-120. URL https://doi.org/10.18799/24131830/2019/1/55 (дата обращения 10.12.2019)

7. A review paper on the application of deconvolution technique in well test analysis: Tal Block Pakistan case study / Muhammad Tauqeer, Safwan Arshad, Zaheer Ahmed, Jawad Ahmed // SPE/PAPG Pakistan section annual technical conference and exhibition. - Pakistan, 2017. https://doi.org/10.2118/191279-MS

8. Optimal control of field development in a closed loop E.S. Zakirov, S.N. Zakirov, I.M. Indrupskiy, O.V. Lyubimova, D.P. Anikeev, I.M. Shiryaev, M.N. Baganova // SPE Russian Petroleum Technology Conference. - Russia, 2015. URL: https://doi.org/10.2118/176642-MS (дата обращения 10.12.2019)

9. ESP-DST well testing in a complex reservoir in the Barents Sea: establishing new methodologies and lessons learned / De Leonardis D., Y. Shumakov, K.L. Morton, S. Sarac // SPE Bergen one day seminar. - Bergen, 2017. URL: https://doi.org/10.2118/185892-MS (дата обращения 10.12.2019).

10. Альберт А. Регрессия, псевдоинверсия и рекуррентное оценивание. - М.: Наука, 1977. - 224 с

11. Robust identification and feedback design: an active noise control case study / R.S. Sánchez Peña, M.A., Cugueró, A. Masip, J. Quevedo, V. Puig // Control Engineering Practice. - 2008. V. 16. - Iss. 11. - P. 1265-1274.

12. Valuable improvements of data preparation and controlling operational regime practices at mature field as a basic part of a smart field concept usage case study / D. Kyrnaev, V. Fomin, A. Maslanov, V. Karpov, A. Amirov, D. Sleptsov, I. Batilov, K. Ratanov, A. Kozhin, A. Klemba, A. Rybalko, M. Guletzskiy // Offshore technology conference Asia. - Kuala Lumpur, 2018. URL: https://doi.org/10.4043/28293-MS (дата обращения 10.12.2019)

13. Автоматизация сбора и подготовки данных (в)ТМС для проведения гидродинамических исследований скважин с использованием «виртуального расходомера» / А.А. Пашали, М.А. Александров, А.Г. Климентьев, И.Р. Ямалов, А.С. Топольников, А.В. Жонин, А.В. Колонских, В.Г. Михайлов // Нефтяное хозяйство. - 2016. - № 11. - С. 60-63.

14. Ипатов А.И., Кременецкий М.И. Геофизический и гидродинамический контроль разработки месторождений углеводородов. - М.: НИЦ «Регулярная и хаотичная динамика», 2006. $780 \mathrm{c}$.

15. Соловьёв И.Г. Структурно-функциональный анализ системы оптимального управления для осложнённого фонда скважин с ЭЦН // НТЖ «Автоматизация, телемеханизация и связь в нефтяной промышленности». - 2019. - № 8. - С. 14-19.

16. Вычислительный анализ режимов эксплуатации скважины с ЭЦН в условиях действия газового фактора / С.С. Белашевский, Д.А. Говорков, Д.В. Житов, И.Г. Соловьев // Автомати- зация, телемеханизация и связь в нефтяной промышленности. 2018. - № 8. - C. 26-30.

17. Сергеев В.Л., Нгуен Т.Х.Ф. Модели и алгоритмы адаптивной интерпретации результатов комбинированных газогидродинамических исследований интеллектуальных скважин // Известия Томского политехнического университета. Инжиниринг георесурсов. - 2018. - Т. 329. - № 10. - С. 67-75.

18. Каталог погружного оборудования УЭЦН TPS-Line (REDA). https://www.slb.ru/upload/iblock/8c4/tps_line_esp-booklet_rus.pdf

19. Технический каталог. Погружное оборудование для добычи нефти и комплексный сервис. URL: https://www.rimera.com/upload/catalog/pdf/Alnas-rus.pdf (дата обращения: 03.10.2019).

20. Подземная гидравлика / К.С. Басниев, А.М. Власов, И.Н. Кочина, В.М. Максимов. - М.: Недра, 1986. - 303 с.

21. Растригин Л.А. Системы экстремального управления. - М.: Наука. Физматлит, 1974. - 632 с.

22. Брилл Дж., Мукерджи Х. Многофазный поток в скважинах. М.; Ижевск: Институт компьютерных исследований, 2006. $384 \mathrm{c}$.

23. Рао С.Р. Линейные статистические методы и их применения. М.: Наука. Физматлит, 1968. -548 с.

24. Соловьев И.Г., Говорков Д.А. Факторы устойчивости МНКоценок параметров модели притока вертикальной скважины // НТЖ Автоматизация, телемеханизация и связь в нефтяной промышленности. - 2009. - № 9. - С. 31-36.

25. Андреев Ю.Н. Управление линейными конечномерными объектами. - М.: Наука. Физматлит, 1976. - 424 с

26. Павлов Б.В., Соловьёв И.Г. Системы прямого адаптивного управления. - М.: Наука, 1989. - 136 с.

27. Luenberger D.G. An introduction to observers // IEEE Transactions on Automatic Control. - 1971. - V. 16 - P. 596-602.

28. Коровин С.К., Фомичёв В.В. Наблюдатели состояния для линейных систем с неопределённостью. - М.: Наука. Физматлит, 2007. -224 с.

29. Fomin V., Solovyev I. Adaptive observer design for hydrodynamic states of an oil-well // Proceeding of CSCS. - Bucharest, 2011. P. 286-289.

30. Nikolov N.N., Lukov V.Y., Alexandrova M.I. Discrete adaptive real-time state observer development using least-squares method // XXVI International Scientific Conference Electronics. - Sozopol, 2017. - P. 1-4.

31. MATLAB - высокоуровневый язык технических расчетов. URL: https://matlab.ru/products/matlab (дата обращения: 03.10.2019)

32. Катковник В.Я. Непараметрическая идентификация и сглаживание данных. - М.: Наука. Физматлит, 1985. - 336 с.

33. Залоруйко Н.Г. Прикладные методы анализа данных и знаний. - Новосибирск: Изд-во Ин-та математики, 1999. - 270 с.

34. Schoukens J., Rolain Y., Pintelon R. On the use of parametric and non-parametric noise-models in time- and frequency domain system identification // 49th IEEE Conference on Decision and Control (CDC). - Atlanta, 2010. - P. 316-321.

35. Сергеев В.Л., Донг Ван Хоанг, Фам Динь Ан. Адаптивная интерпретация результатов гидродинамических исследований горизонтальных скважин на прогнозирующих моделях // Известия Томского политехнического университета. Инжиниринг георесурсов. - 2019. - Т. 330. - № 1. - С. 165-172.

Поступила 05.05.2020 2.

\section{Информация об авторах}

Соловьев И.Г., кандидат технических наук, доцент, старший научный сотрудник, Федеральный исследовательский центр Тюменский научный центр СО РАН; доцент, Тюменский индустриальный университет.

Говорков Д.А., кандидат технических наук, старший научный сотрудник, Федеральный исследовательский центр Тюменский научный центр СО РАН; доцент, Тюменский индустриальный университет.

Цибульский В.P., доктор технических наук, профессор, ведущий научный сотрудник, Федеральный исследовательский центр Тюменский научный центр СО РАН. 
UDC 622.279:681.5

\title{
IDENTIFICATION OF A WELL HYDRODYNAMIC MODEL WITH AN ELECTRIC SUBMERSIBLE PUMP USING THE PERTURBED OPERATION MODES CONTROL DATA
}

\author{
llya G. Solovyev 1,2 , \\ solovyev@ikz.ru
}

\section{Denis A. Govorkov1,2,} dagovorkov@mail.ru

Vladimir R. Tsibulsky ${ }^{1}$,
v-tsib@yandex.ru

1 Tyumen Scientific Centre SB RAS,

86, Malygin street, Tyumen, 625000 , Russia.

2 Tyumen Industrial University, 38 , Volodarsky street, Tyumen, 625000 , Russia.

The relevance of the research is associated with the problems of creating and maintaining dynamic models of electrical submerged pumpequipped well as a part of operational monitoring and control systems under conditions of the elevator and inflow parameters deviation. Assessment of the root causes of well desired operating mode violation and the forecast of the immediate negative consequences are important for balanced (optimal) decisions on operational modes correction and ensuring stable operating conditions.

The main aim of the research is to develop the least-squares algorithm - estimating the drift parameters of the elevator including the electrical submerged pump and the inflow of a dynamic well model and methods for stabilizing these estimates in real-time operations based on noisy measurements data.

Methods: numerical integration of nonlinear differential equations, regression least-squares analysis methods, statistical computational tests, test perturbations, methods of regularizing estimation algorithms.

Results. The authors have developed the two-stage algorithm for estimating the drift parameters of the elevator (wear, impurities) and inflow (productivity and reservoir parameters) to simplify the presentation of a complex dynamic model «inflow-elevator-electrical submerged pump-wellhead» according to two speed scales for monitoring the operating conditions of the system with inflow dynamics observer under conditions of test frequency disturbances.

Summary. The proposed rules for stabilizing the model parameters estimates based on the generation of test perturbations, reducing dimension by decomposing the complex model into composite blocks, filtering noisy measurement signals by approximation, implementing a dynamic inflow observer form the basis for identification algorithm design with reliable estimation of the main group of deformation parameters of pump pressure-head characteristics and inflow. Despite the low accuracy of the elevator hydraulic resistance identification, the error in reproducing the dynamics of the variable states of the system in disturbed operating modes from the observer outputs does not exceed $2 \%$, which indicates the low sensitivity of the standard operating modes of the elevator to the desired parameter setting within the framework of the model solutions used. The required accuracy of the pipe hydraulic resistance identification should be consistent with the conditions for the periodic cleaning of the lift from sorbents.

\section{Key words:}

Well, electric centrifugal pump, complicating factors, decomposition, regularization, assessment, identification, least squares method, observers, state filters, test disturbances.

\section{REFERENCES}

1. Hashim Al-Sadah, Mohannad Abo Khamseen, Abdullah AlGhamdi, Ali Fardan. Proactive utilization of ESP performance monitoring to enhance productivity. SPE middle east oil and gas show and conference. Bahrain, 2019. Available at: https://doi.org/10.2118/194925-MS (accessed 10 December 2019).

2. Lichi Deng, Ehsan Davani, Hamed Darabi, Vural Sander Suicmez, David Castineira. Rapid and comprehensive artificial lift systems performance analysis through data analytics, diagnostics and solution evaluation. SPE middle east artificial conference and exhibition. Bahrain, 2018. Available at: https://doi.org/10.2118/192460MS (accessed 10 December 2019).

3. Mriganga Das, Zadil Hayat Bin Naharuddin, Dhuha Al-Khalid Optimizing production and operation through automated well and network modeling. SPE Kuwait oil and gas show and conference. Kuwait, 2015. Available at: https://doi.org/10.2118/175205-MS (accessed 10 December 2019).

4. Reda El Mahbes, Wilfried Manfoumbi, Benedicte Kadio-Morokro. Real-time remote monitoring to enhance electrical submersible pump system run life and maximize production. SPE middle east artificial lift conference and exhibition. Bahrain, 2018. Available at: https://doi.org/10.2118/192470-MS (accessed 10 December 2019).
5. Gabor Takacs. Three inventions shaping the future of ESP technology. Journal of Petroleum Science and Engineering, 2019, vol. 182. Available at: https://doi.org/10.1016/j.petrol.2019.106330 (accessed 10 December 2019).

6. Solovyev I.G., Govorkov D.A., Belashevsky S.S., Vedernikova U.A. Transient processes model of well with frequency-regulated ESP. Bulletin of the Tomsk Polytechnic University. Geo Assets Engineering. 2019. vol. 330. No 1. pp. 110-120. In Rus. Available at: https://doi.org/10.18799/24131830/2019/1/55 (accessed 10 December 2019).

7. Muhammad Tauqeer, Safwan Arshad, Zaheer Ahmed, Jawad Ahmed. A review paper on the application of deconvolution technique in well test analysis: Tal Block Pakistan case study. SPE/PAPG Pakistan section annual technical conference and exhibition. Pakistan, 2017. Available at: https://doi.org/10.2118/191279-MS (accessed 10 December 2019).

8. Zakirov E.S., Zakirov S.N., Indrupskiy, I.M. Lyubimova O.V., Anikeev D.P., Shiryaev I.M., Baganova M.N. Optimal Control of Field Development in a Closed Loop. SPE Russian Petroleum Technology Conference. Moscow, 2015. Available at: https://doi.org/10.2118/176642-MS (accessed 10 December 2019).

9. De Leonardis D., Shumakov Y., Morton K.L., Sarac S. ESP-DST well testing in a complex reservoir in the Barents Sea: establishing 
new methodologies and lessons learned. SPE Bergen one day seminar. Bergen, Norway, 5 April 2017. Available at: https://doi.org/10.2118/185892-MS (accessed 10 December 2019).

10. Albert A. Regressiya, psevdoinversiya i rekurrentnoe otsenivanie [Regression, pseudo-inversion and recurrence estimation]. Moscow, Nauka Publ., 1977. 224 p.

11. Sánchez Peña R.S., Cugueró M.A., Masip A., Quevedo J., Puig V. Robust identification and feedback design: An active noise control case study. Control Engineering Practice. 2008, vol. 16, Iss. 11, pp. $1265-1274$

12. Kyrnaev D., Fomin V., Maslanov A., Karpov V., Amirov A. Sleptsov D., Batilov I., Ratanov K., Kozhin A., Klemba A., Rybalko A., Guletzskiy M. Valuable improvements of data preparation and controlling operational regime practices at mature field as a basic part of a smart field concept usage case study. Offshore technology conference Asia. Kuala Lumpur, 2018. Available at: https://doi.org/10.4043/28293-MS (accessed 10 December 2019).

13. Pashali A.A., Aleksandrov M.A., Klimentev A.G., Yamalov I.R., Topolnikov A.S., Zhonin A.V., Kolonskih A.V., Mikhaylov V.G. Automatization of collecting and preparation of telemetry data for well testing using «virtual flowmeter». Oil industry, 2016, no. 11, pp. 60-63. In Rus.

14. Ipatov A.I., Kremenetskiy M.I. Geofizicheskiy i gidrodinamicheskiy kontrol razrabotki mestorozhdeniy uglevodorodov [Geophysical and hydrodynamic control of hydrocarbon field development Moscow, Regulyarnaya i khaotichnaya dinamika Publ., 2006. $780 \mathrm{p}$.

15. Solovyev I.G. Structural-functional analysis of the optimal contro system for complicated fund of wells with ESP. ScientificTechnical Journal «Automation, telemechanization and communication in oil industry, 2019, no. 8, pp. 14-19. In Rus.

16. Belashevskiy S.S., Govorkov D.A., Solovyev I.G. Periodical service regulation of complicated wells. Scientific-Technical Journal «Automation, telemechanization and communication in oil industry, 2018, no. 1, pp. 21-26. In Rus.

17. Sergeev V.L., Phuong T.H. Nguyen. Models and algorithms for adaptive interpretation of combined well test data of intelligen wells. Bulletin of the Tomsk Polytechnic University. Geo Assets Engineering, 2018, vol. 329, no. 10, pp. 67-75. In Rus.

18. Katalog pogruzhnogo oborudovaniya UECN TPS-Line (REDA) [Catalog of submersible equipment of ESP]. Available at: https://www.slb.ru/upload/iblock/8c4/tps_line_esp-booklet_rus.pdf (accessed 10 December 2019)

19. Tekhnicheskiy katalog. Pogruzhnoe oborudovanie dlya dobychi nefti i kompleksny servis [Technical catalog. Submersible equipment for oil production and comprehensive service.] Available at: https://www.rimera.com/upload/catalog/pdf/Alnas-rus.pdf $\quad$ (accessed 3 October 2019)

20. Basniev K.S., Vlasov A.M., Kochina I.N., Maksimov V.M. Podzemnaya gidravlika [Underground hydraulics]. Moscow, Nedra Publ., 1986. 303 p.

\section{Information about the authors}

Ilya G. Solovyev, senior researcher, Tyumen Scientific Centre SB RAS; associate professor, Tyumen Industrial University.

Denis A. Govorkov, senior researcher, Tyumen Scientific Centre SB RAS; associate professor, Tyumen Industrial University.

Vladimir R. Tsibulsky, lead researcher Tyumen Scientific Centre SB RAS.
21. Rastrigin L.A. Sistemy ekstremalnogo upravleniya [Extreme control systems]. Moscow, Nauka Fizmatlit Publ., 1974. 632 p.

22. Brill Dzh., Mukerdzhi Kh. Mnogofazny potok v skvazhinakh [Multiphase flow in wells]. Moscow; Izhevsk, Computer research institute Publ., 2006. 384 p.

23. Rao S.R. Lineynye statisticheskie metody i ikh primeneniya [Linear statistical methods and their applications]. Moscow, Nauka. Fizmatlit. Publ., 1968. 548 p. Journal «Automation, telemechanization and communication in oil industry, 2009, no. 9, pp. 31-36. In Rus.

25. Andreev Yu.N. Upravlenie lineynymi konechnomernymi obyektami [Management of linear finite-dimensional object]. Moscow, Nauka. Fizmatlit. Publ., 1976. 424 p.

26. Pavlov B.V., Solovyov I.G. Sistemy pryamogo adaptivnogo upravleniya [Direct adaptive control systems]. Moscow, Nauka Publ., 1989. $136 \mathrm{p}$.

27. Luenberger D.G. An introduction to observers. IEEE Transactions on Automatic Control, 1971, Vol. 16, pp. 596-602.

28. Korovin S.K., Fomichov V.V. Nablyudateli sostoyaniya dlya lineynkh sistem s neopredelennostyu [State observer for linear systems with uncertainty]. Moscow, Nauka. Fizmatlit. Publ., 2007. $224 \mathrm{p}$.

29. Fomin V., Solovyev I. Adaptive observer design for hydrodynamic states of an oil-well. Proceeding of CSCS -18. Bucharest, 2011. pp. 286-289.

30. Nikolov N.N., Lukov V.Y., Alexandrova M.I. Discrete adaptive real-time state observer development using least-squares method. XXVI International Scientific Conference Electronics. Sozopol, 2017. pp. $1-4$

31. MATLAB. Available at: https://www.mathworks.com/products/ matlab.html (accessed 3 October 2019).

32. Katkovnik V.Ya. Neparametricheskaya identifikatsiya i sglazhivanie dannykh [Nonparametric identification and data smoothing.]. Moscow, Nauka. Fizmatlit. Publ., 1985. 336 p.

33. Zaloruyko N.G. Prikladnye metody analiza dannykh $i$ znaniy [Applied methods of data and knowledge analysis.]. Novosibirsk, Mathematics Institute Publ., 1999. 270 p.

34. Schoukens J., Rolain Y., Pintelon R. On the use of parametric and non-parametric noise-models in time- and frequency domain system identification. $49^{\text {th }}$ IEEE Conference on Decision and Control (CDC). Atlanta, 2010. pp. 316-321.

35. Sergeev V.L., Dong Van Hoang, Pham Dinh An. Adaptive interpretation of the results of horizontal well production testing using forecasting models. Bulletin of the Tomsk Polytechnic University. Geo Assets Engineering, 2019, vol. 330, no. 1, pp. 165-172.

Received: 5 May 2020.
24. Solovyev I.G., Govorkov D.A. Least-sqaure estimations of oil well inflow characteristics: factors of stability. Scientific-Technical 